\title{
The genomics of dopamine agonists treatment of schizophrenia: a case of homozygous valine catechol- o-methyltransferase polymorphism
}

\begin{abstract}
We present the case of a patient with antipsychotics nonresponsive negative symptoms of Schizophrenia who responded significantly to a dopamine agonist within six days of treatment. He was previously unresponsive to other psychotropic medications. Genomic testing of the patient revealed a Homozygous Valine Catechol-O-Methyltransferase (COMT) polymorphism which is associated with high metabolism of dopamine in the frontal cortex with subsequent low dopamine activity. The significance of this finding for selecting good candidates for psychostimulant therapy for negative symptoms is discussed.
\end{abstract}

Keywords: schizophrenia, paliperidone, aripiprazole
Volume II Issue 2 - 2020

\author{
Puja Patel,' Vatsala Sharma, ${ }^{2}$ Tasmia Khan, ${ }^{3}$ \\ George Letterio, ${ }^{3}$ Ravindi Gunasekara, ${ }^{3}$ \\ Gurtej Singh Gill, ${ }^{4}$ Samuel Adeyemo, ${ }^{4}$ Heela \\ Azizi,' Jesslin Abraham,' Olusegun Popoola, ${ }^{4}$ \\ Chiedozie Ojimba, ${ }^{4}$ Karthik Cherukupally, ${ }^{4}$ \\ Olaniyi Olayinka, ${ }^{4}$ Ayodeji Jolayemi ${ }^{4}$ \\ 'Department of Psychiatry, Interfaith Medical Center,American \\ University of Antigua College of Medicine, USA \\ ${ }^{2}$ Extern, Department of Psychiatry, Interfaith Medical Center, \\ USA \\ ${ }^{3}$ Department of Psychiatry, Interfaith Medical Center, Medical \\ University of the Americas, USA \\ ${ }^{4}$ Department of Psychiatry, Interfaith Medical Center, USA
}

\author{
Correspondence: Puja Patel, Department of Psychiatry, \\ American University of Antigua College of Medicine, Interfaith \\ Medical Center, Brooklyn, New York, USA, \\ Email pujapa@auamed.net
}

Received: May 08, 2020 | Published: May 28, 2020

\section{Introduction}

The negative symptoms of Schizophrenia are defined as diminished emotional expression and avolition. ${ }^{1}$ Alogia, anhedonia, asociality are few other symptoms mentioned in the DSM $5 .^{2}$ The Management of negative symptoms remains unclear and has proven difficult. Atypical antipsychotics such as olanzapine, quetiapine, and newer generation drugs such as paliperidone were used in a few studies based on the understanding we have of the role serotonin plays in negative symptoms. ${ }^{3}$ In a study entitled Cost Utility of the Latest Antipsychotic Drugs in Schizophrenia Study (CUtLASS), Jones et al found that a comparison of typical and atypical antipsychotics showed no significant difference in effectiveness between a typical and an atypical antipsychotic drug. ${ }^{4}$ Another treatment proposal was the use of antidepressants, which was also found to have insufficient evidence for use in management. ${ }^{5}$

The poor response of negative symptoms of Schizophrenia to antipsychotics and antidepressants have led to the study of psychostimulants. The choice of psychostimulants is based on the understanding that negative symptoms were caused by dopaminergic hypoactivity in the mesocortical pathway and prefrontal cortex. A study conducted by Lindenmayer et al showed an overall improvement of $37 \%$ to $67 \%$ in negative symptom score using adjunct dopamine agonists. ${ }^{6}$ However, these studies also lacked an appropriate sample size. The characterization of the types of patients who responded to psychostimulants was not also made in the study.

It is for this reason that we present the case of a patient with antipsychotic nonresponsive negative symptoms of Schizophrenia who responded significantly to stimulants within six days of treatment. We investigated factors that may have led to his rapid favorable improvement. characterized his genetic profile specifically Homozygous Valine Catechol-O-Methyltransferase (COMT). This polymorphism is associated with high metabolism of dopamine in the frontal cortex with subsequent low dopamine activity in this region. The significance of these findings in the treatment of negative symptoms of Schizophrenia with psychostimulants are discussed including the selection of patients for psychostimulant studies possibly based on COMT polymorphism.

\section{Case presentation}

We present the case of a 41-year-old man with a prior psychiatric history of multiple inpatient admissions for a diagnosis of Schizophrenia and comorbid diagnosis of Hypertension, Diabetes Mellitus, Alcohol Use Disorder and Cannabis Use Disorder. He was brought to the psychiatric emergency room by family members, for increasing disorganization of behavior and thought, with associated poor self care and social withdrawal in the context of noncompliance with his antipsychotic medications. There was no report of illicit substance use. He appeared unkempt, malodorous and emotionally withdrawn. His presentation was notable for severe negative symptoms such as blunted affect, disorganized thought process, disheveled appearance, poor hygiene, monosyllabic and monotonic responses. Positive and Negative Symptoms Scale Assessment (PANSS) was administered which showed a total score of 126, with 38 on Positive, 39 on Negative, and 49 on Global subscale. A description of his score is presented in Table 1 . 
Table I Initial PANSS Score

\begin{tabular}{|c|c|c|c|}
\hline \multicolumn{4}{|c|}{ Positive and negative symptoms scale on admission } \\
\hline Positive score & & General psychopathology score & \\
\hline PI- Delusion & 7 & GI-Somatic concern & I \\
\hline P2- Conceptual Disorganisation & 7 & G2-Anxiety & 3 \\
\hline P3- Hallucinatory behavior & 3 & G3- Guilt Feelings & I \\
\hline P4- Excitement & 1 & G4-Tension & I \\
\hline P5- Grandiosity & 6 & G5- Mannerism and posturing & 4 \\
\hline P6- Suspiciousness/persecution & 7 & G6- Depression & I \\
\hline \multirow[t]{2}{*}{ P7- Hostility } & 7 & G7- Motor Retardation & 6 \\
\hline & & G8- Uncooperativeness & 3 \\
\hline Negative score & & G9-Unusual thought content & 6 \\
\hline NI- Blunted Affect & 7 & GI0- Disorientation & I \\
\hline N2- Emotional Withdrawal & 6 & GII- Poor attention & 3 \\
\hline N3-Poor Rapport & 4 & GI2- Lack of judgement and insight & 6 \\
\hline N4- Passive/apathetic Social Withdrawal & 7 & GI3- Disturbance of Volition & 3 \\
\hline N5-Difficulty in abstract thinking & 4 & GI4- Disturbance of volition & 4 \\
\hline N6-Lack of spontaneity and flow of conversation & 6 & GI5- Preoccupation & 3 \\
\hline N7- Stereotyped thinking & 5 & GI6-Active social avoidance & 3 \\
\hline
\end{tabular}

A review of affective symptoms revealed no depressive or manic syndrome and he scored 7 and 4 on Hamilton Depression Rating Scale and Young Mania Rating Scale, respectively. No suicidal or homicidal ideations were elicited. A mini-mental status examination showed no evidence of disorientation or cognitive impairment. Initial laboratory work for underlying medical causes and in light of his poor self care, demonstrated no objective abnormalities in thyroid function test, complete blood count, with normal white cell count and other parameters of hematology. A comprehensive metabolic profile demonstrated no abnormalities in electrolytes. A slightly elevated blood glucose $(145 \mathrm{mg} / \mathrm{dl})$ and $\mathrm{HbAlc}(7.8)$ was found, which was consistent with his history of diabetes. Urinalysis was normal and urine toxicology did not reveal any evidence of illicit substance use. A routine chest radiology showed no acute signs of infection. A Non Contrast Computerized Tomography (CT) Scan of the brain was normal, with the result shown in Figure 1.

As he was non-compliant with Aripiprazole prior to coming to the hospital because of side effects of Akathisia, he was initiated on Risperidone $4 \mathrm{mg}$ daily and was titrated to $8 \mathrm{mg}$ daily by the ninth day. His Positive and Negative Symptoms Scale Assessment (PANSS) showed a total score of 115, with 27 on Positive, 39 on Negative, and 49 on Global subscale. No improvement in negative symptoms subscale was noted.

Based on the poor response of negative symptoms, Clozapine augmentation was started at the dose of $50 \mathrm{mg}$ daily of Clozapine on day 15 which was gradually increased to $100 \mathrm{mg}$ daily by Day 22 . However, the patient's Absolute Neutrophil Count (ANC) dropped to 1.1 from the baseline of 3.9 , which necessitated the discontinuation of Clozapine. Loxapine as an augmentation for Risperidone was subsequently initiated, in place of Clozapine, with a dose of $100 \mathrm{mg} /$ day of Loxapine achieved by day 29. The patient continued to exhibit poor self care, reduced psychomotor activity, asociality, inattention, as well as poor insight and judgement. The antipsychotics were continued in the same dose over the next 7 days. His Positive and Negative Symptoms Scale Assessment (PANSS) showed a total score of 111, with 23 on Positive, 39 on Negative, and 49 on Global subscale. His negative symptoms showed no improvement.

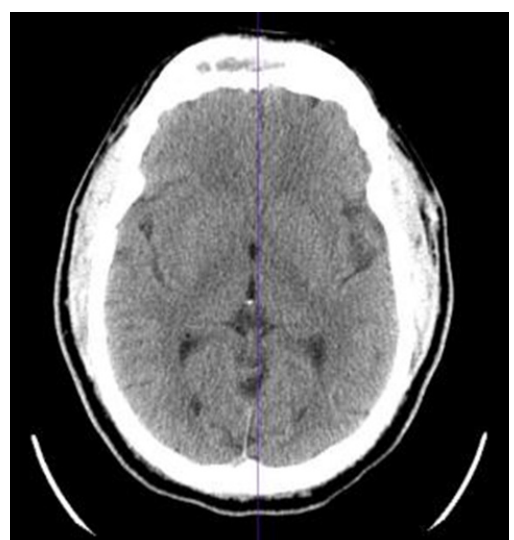

Figure I Transverse view of head CT was unremarkable.

Due to the patient's persistent negative symptoms and poor response to antipsychotic medications, a pharmacogenetic profile testing for medication selection was conducted. An oral swab was obtained 
for DNA samples, and an assay using a technique called real-time Polymerase Chain Reaction (PCR) was performed on the samples. The technique tested for the presence of specific gene variants which have been linked with particular symptomatic profile and response to antipsychotic medications. Sixty of these gene locations (also called

Table 2 Pharmacogenetic test results
Single Nucleotide Polymorphisms or SNPs) were tested for each patient sample. The results of the genetic test showed a Catechol-OMethyltransferase Polymorphism that was the Homozygous variant and associated with rapid metabolism of Dopamine neurotransmitter (Table 2). ${ }^{7}$

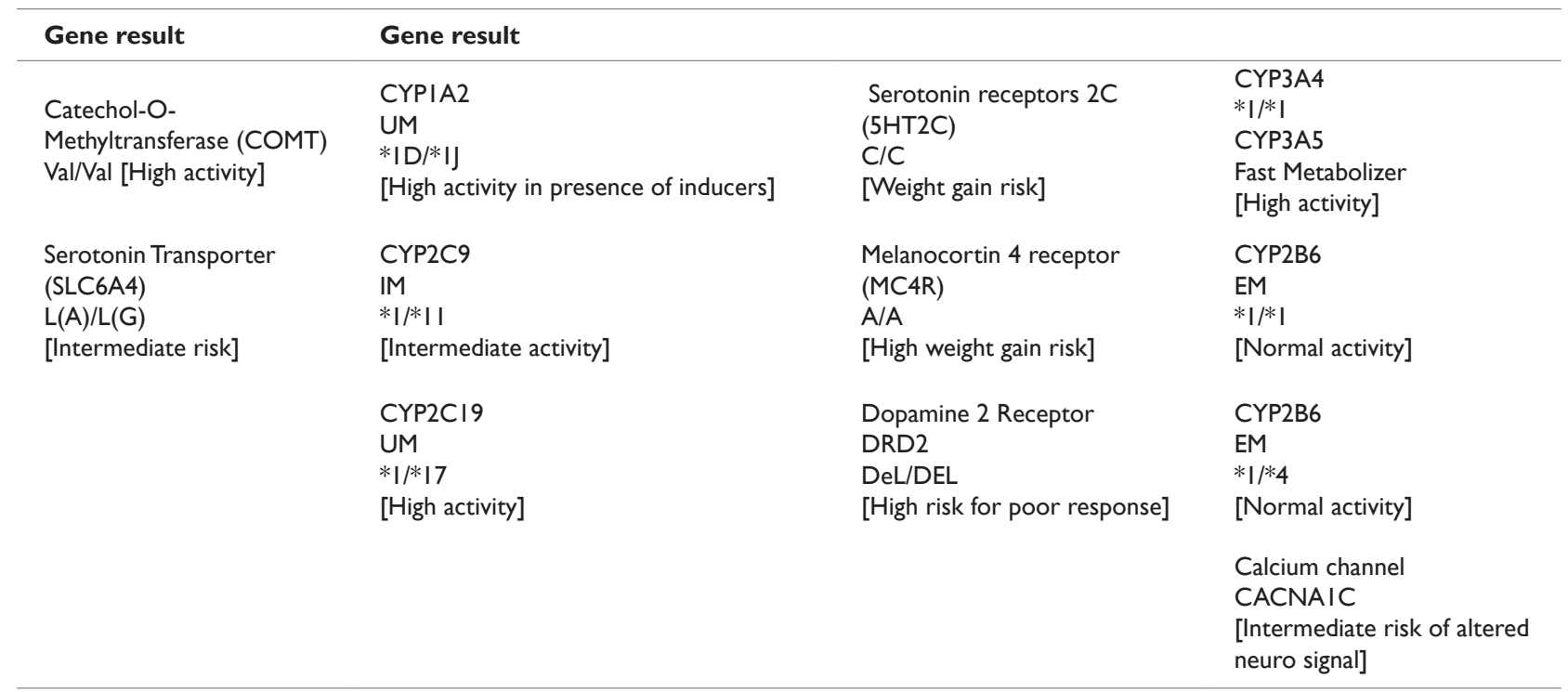

An assessment was made that the rapid metabolism of dopamine likely predisposed him to developing the negative symptoms, and a dopamine agonist Amantadine was initiated as an augmentation for Risperidone and Loxapine. The initial PANSS score on the day of initiation on Amantadine was 18 on Positive Subscale, 39 on Negative Subscale and 44 on the subscale of General Psychopathology. Amantadine was started in the dose of $100 \mathrm{mg}$ daily and was increased to $200 \mathrm{mg} /$ day over four days. And continued at $200 \mathrm{mg}$ per day. He received a course of Amantadine for a total of six days. He showed a dramatic improvement of a 19 point drop in negative symptoms, over the six days of Amantadine treatment. His positive symptoms did not worsen as a result of augmentation with Amantadine, but instead showed steady improvement. A tabulated form of his improvement on medications is shown in Table 3 .

Table 3 Total PANSS Scores

\begin{tabular}{lllll}
\hline PANSS & $\begin{array}{l}\text { First day amantadine } \\
\text { augmentation }\end{array}$ & $\begin{array}{l}\text { Fourth day } \\
\text { amantadine } \\
\text { augmentation }\end{array}$ & $\begin{array}{l}\text { Fifth day } \\
\text { amantadine } \\
\text { augmentation }\end{array}$ & $\begin{array}{l}\text { Sixth day amantadine } \\
\text { augmentation }\end{array}$ \\
\hline Total Score & 103 & 60 & 50 & 49 \\
Positive & 18 & 7 & 7 & 7 \\
Negative & 39 & 22 & 21 & 20 \\
Global & 44 & 31 & 22 & 22 \\
\hline
\end{tabular}

It was noticed that the patient's emotional reactivity improved and showed good eye-contact. He used much more complete sentences and his response latency decreased significantly. The patient's overall grooming and hygiene remained suboptimal. The patient was subsequently discharged home on Loxapine 100mg, Risperidone $4 \mathrm{mg}$ and Amantadine $200 \mathrm{mg}$ daily. He was motivated to engage in outpatient treatment. He exhibited goal directed activities and was interested in performing instrumental activities of daily living.

\section{Discussion}

The patient presented with an acute psychotic exacerbation of Schizophrenia, with a mixture of positive and negative symptoms. The effect of underlying medical causes and illicit substances was ruled out by normal laboratory findings. A PANSS assessment was able to provide a characterization of the severity of positive and negative symptoms and provide a baseline to assess the effectiveness of treatment. He was initiated on antipsychotics with marginal improvement on Risperidone, necessitating the augmentation with Clozapine. There is evidence that negative symptoms, in association with positive symptoms respond well to clozapine. ${ }^{8}$

Clozapine was soon discontinued due to decreased absolute neutrophil count (dropped to 1.1 from a baseline of 3.9). The decreased absolute neutrophil count was a concern due to one of the severe side effects of clozapine: agranulocytosis. Loxapine was initiated as a substitute for Clozapine due to its similarities in characteristics. The chemical structure of Loxapine is similar to Clozapine. Loxapine 
acts like an atypical antipsychotic due to its binding properties at dopamine-2 (D2), serotonin-2A (5-HT2A) receptors and high 5-HT2/ D2 ratio. Loxapine is a more potent 5-HT2A antagonist and like Clozapine, it binds with higher affinity to D4 receptors as compared to other dopaminergic receptors. ${ }^{9}$ The patient's negative symptoms however showed no improvement on antipsychotics. This observation is supported by CUtLASS which proposed that as opposed to First Generation Antipsychotics, there is no advantage in terms of quality of life, symptoms or costs of care across 1 year in commencing treatment with atypical Second Generation Antipsychotics. ${ }^{4}$

In the absence of significant improvement, pharmacogenetic characterization for targeted treatment was explored. The results of pharmacogenetic testing showed a Homozygous Valine variant Catechol-O-Methyltransferase Polymorphism that results in high metabolism of dopamine in the frontal cortex. As negative symptoms are due to reduced dopaminergic activity in the mesocortical region ${ }^{10}$ dopamine agonist could serve as potential treatments for patients with negative symptoms. In our patient, Amantadine was selected as the dopamine agonist of choice. He showed improvement over six days of Amantadine administration with PANSS Negative subscale drop from 39 on to 20 . Amantadine did not cause deterioration of the positive symptoms in our patient.

The mechanism of action of Amantadine is complex. It does not directly interact with dopamine receptors but has indirect dopaminomimetic actions by enhanced dopamine release and turnover through NMDA antagonistic

properties. ${ }^{11}$ NMDA receptors are hypofunctional in Schizophrenia patients which could lead to a compensatory excessive glutamate release in an attempt to overcome the deficit. So, reversing this pattern with the antagonists may be helpful in reducing schizophrenia symptoms. ${ }^{12}$

The dopaminergic action of amantadine by its effect on NMDA receptors can be explained broadly via several mechanisms including: stimulation of L-glutamate release (which is modulated by activation of cystine/glutamate antiporter (Sxc) in both cognition generator regions, the entorhinal cortex and globus pallidus), enhancement of glutathione synthesis via Sxc activation and inhibition of ionotropic glutamate receptors (NMDA receptors) ${ }^{13}$ So, activation of Sxc increases the L-glutamate release but NMDA Receptor antagonism prevents the neurotoxic response induced by Sxc. A combination of inhibition of NMDA Receptors and partial activation of Sxc, contributes to the actions of Amantadine. ${ }^{13}$

This report on improvement of negative symptoms with a dopamine agonist adds to literature on the use of stimulants for managing negative symptoms of Schizophrenia. Several studies have also reported results of different stimulants on the negative symptoms of schizophrenia. One of which is a multicenter study conducted by Lasser et al, the stimulant lisdexamfetamine dimesylate (LDX) was given as an adjunctive therapy to antipsychotics in adults with clinically stable schizophrenia who predominantly had negative symptoms of schizophrenia. Patients went through 10-week openlabel adjunctive LDX, and a 4-week, double-blinded, randomized, placebo-controlled withdrawal. The open-label LDX trial appeared to be associated with significant improvements in negative symptoms, however, the double-blinded / randomized withdrawal phase showed no significant differences found between the placebo and LDX in SANS-18 or PANSS subscale scores. ${ }^{3}$
In a study by Tamminga et al. ${ }^{14}$ the effects of N-propylnorapomorphine, an aporphine-family dopamine (DA) agonist was explored in schizophrenic patients with active psychotic symptoms. Results from this study supported the idea that the acute antipsychotic response of DA agonists is mediated by the DA autoreceptor. N-propylnorapomorphine alter brain mesencephalic DA systems by reducing DA-mediated neural transmission. ${ }^{14}$

As our case demonstrated improvement over a short period of six days, further studies are required to monitor the long term effect of Amantadine on the maintenance of therapeutic effect on the negative symptoms. In addition, studies are needed to explore the possibility of development of positive symptoms in the long run. In addition, it should be noted that our case is of dramatic improvement in a patient with Homozygous Valine variant of Catechol-O- Methyltransferase Polymorphism. It is not clear if similar improvements will be seen in patients with other genotypes and further studies are needed to explore this. This is especially important given the reports mentioned with variable response to stimulant treatment in patients with positive and negative symptoms of Schizophrenia.

\section{Conclusion}

Amantadine may be of short term clinical benefit for negative symptoms of Schizophrenia unresponsive to antipsychotic medications. This may especially be the case in a patient with Homozygous Valine variant of Catechol-O-Methyltransferase Polymorphism. Further studies are needed to explore long term therapeutic benefits of Amantadine for negative symptoms of Schizophrenia, as well as other genotypes of patients responsive to dopamine agonist.

\section{Acknowledgments}

None.

\section{Conflicts of interest}

The authors have no conflicts of interest to declare.

\section{Authors contributions}

All authors have participated in the procurement of this document and agree with the submitted case report.

\section{References}

1. American Psychiatric Association. Diagnostic and Statistical Manual of Mental Disorders. 5th edn. Washington DC: American Psychiatric Association; 2013.

2. Lindenmayer JP, Nasrallah H, Pucci M, et al. A systematic review of psychostimulant treatment of negative symptoms of schizophrenia: Challenges and therapeutic opportunities. Schizophr Res. 2013;147(2):241-252.

3. Lasser RA, Dirks B, Nasrallah H, et al. Adjunctive lisdexamfetamine dimesylate therapy in adult outpatients with predominant negative symptoms of schizophrenia: open-label and randomized-withdrawal phases. Neuropsychopharmacology. 2013;38(11):2140-2149.

4. Jones PB, Barnes TR, Davies L, et al. Randomized controlled trial of the effect on Quality of Life of second-vs first-generation antipsychotic drugs in schizophrenia: Cost Utility of the Latest Antipsychotic Drugs in Schizophrenia Study (CUtLASS 1). Archives of general psychiatry. 2006;63(10):1079-1087. 
5. Remington $\mathrm{G}$, Foussias $\mathrm{G}$, Fervaha $\mathrm{G}$, et al. Treating negative symptoms in schizophrenia: an update. Current treatment options in psychiatry. 2016;3(2):133-150.

6. Leucht S, Cipriani A, Spineli L, et al. Comparative efficacy and tolerability of 15 antipsychotic drugs in schizophrenia: A multiple-treatments metaanalysis. Lancet. 2013;382:951-962.

7. Wang Y1, Fang Y, Shen Y, et al. Analysis of association between the catechol -O- methyltransferase (COMT) gene and negative symptoms in chronic schizophrenia. Psychiatry Res. 2010;179(2):147-150.

8. Khan AH, Zaidi S. Clozapine: improvement of negative symptoms of schizophrenia. Cureus. 2017;9(12):e973.

9. Popovic D, Nuss P, Vieta E. Revisiting loxapine: a systematic review. Annals of general psychiatry. 2015;14(1):15.
10. Downar J, Kapur S. Biological Theories. In: Mueser KT, Jeste DV, Editors. Clinical handbook of schizophrenia. Guilford Press; 2011:25-34.

11. Transm JN. Amantadine and memantine are NMDA receptor antagonists with neuroprotective properties. J Neural Transm. 1994;43:91-104.

12. Stahl S. Antipsychotic agents, in Stahl's Essential Psychopharmacology. 3rd edn, Cambridge University Press; 2008. pp. 440-446.

13. Nakano T, Hasegawa T, Suzuki D, et al. Amantadine Combines Astroglial System Xc-Activation with Glutamate/NMDA Receptor Inhibition. Biomolecules. 2019;9(5):191.

14. Tamminga CA, Gotts MD, Thaker GK, et al. Dopamine Agonist Treatment of Schizophrenia With N-Propylnorapomorphine. Arch Gen Psychiatry. 1986;43(4):398-402. 